\title{
CELESTIAL REFERENCE FRAMES
}

\author{
J. Kovalevsky \\ C.E.R.G.A. Grasse, France
}

ABSTRACT. A celestial reference frame is based on some dynamical or kinematic approximation of an absolute coordinate system and is materialized by a fundamental catalogue of stars. It is characterized by the accuracy of the system and the precision of its stellar realization.

The present situation, marked by the construction of the FK5 system and catalogue, is briefly described. It will be an hybrid system based on the discussion of all existing observations. The prospects for improvement in accuracy are good using lunar laser observations or VLBI determination of the positions of extra-galactic radio-sources. Three procedures that may be used to link such a system to a star catalogue are given. A major improvement in precision is expected from the lipparcos programme and further extensions include essentially the densification of catalogues with stars of magnitudes 10 to 14 using photoelectric automatic meridian instruments.

\section{CONCEPTUAL BACKGROUND}

The purpose of a celestial reference frame is to provide a materialization of a coordinate system in order to describe various motions observed in the Earth-Moon system, the solar system and, more generally, anywhere in the Universe. No motion can validly be studied unless it is referred by a known process to some coordinate system in which one can write the dynamical equations of motion. This means that the observations must be made in such a way that they can be expressed in terms of coordinates in this system. For instance, in the case of celestial reference frames, the observations are made with respect to a stellar background, the stars providing the materialization of the coordinate system.

Conceptua11y, the simplest reference system is inertial: There, the differential equations of motion may be written without including any rotational term. Such a system is dynamical in character and can be constructed uniquely from the analysis of the motion of one or several celestial bodies such as the Moon or the planets, provided that all

\section{7}

E. M. Gaposchkin and B. Kotaczek (eds.), Reference Coordinate Systems for Earth Dynamics, 77-86. Copyright $\odot 1981$ by D. Reidel Publishing Company. 
interacting forces and initial conditions are correctly evaluated. This means that a correct model of the dynamical system must exist (Kovalevsky, 1975 b). This is by itself a major difficulty that has been described by Mulholland (1977) for the lunar motion or by Duncombe et al. (1975) for planetary motions. But another difficulty of a purely dynamical reference frame is that, strictly speaking, its availability for the observation of a given body implies a simultaneous observation of at least one of the bodies used in the definition of the system. Except in some specific cases, this is not convenient. Thus, it is necessary to have the positions and motions of many more bodies in this frame and use them as fiducial points that materialize it. If, as is usually the case, these points are stars, we have a celestial reference frame defined dynamically. The list of stars, with their positions at epoch and their proper motions referred to the frame constitutes the fundamental catalogue materializing the celestial reference frame.

Another, actually simpler, definition of a celestial reference frame is found if the defining bodies are sufficiently remote so that they can be considered as fixed or slowly moving in the sky in a modellable fashion. These stars or extragalactic objects also serve as the materialization of the celestial frame. The latter is, in this case, defined kinematically. But again, their number may be insufficient for many purposes and it is again necessary to increase it by adding coordinates and proper motions of secondary stars in quite a similar manner as in the case of a dynamically defined celestial system.

The existence of two theoretically - if not always practically separated concepts in a celestial reference system has led us to separate also the concepts of the precision with which their realization represents reality: Let us, therefore, propose two definitions:

(a) The accuracy of a celestial reference frame is the evaluation of the error of the dynamical or kinematic definition of the frame, due to errors in the modelling or in the determination of kinematic conditions. If the construction of the catalogue introduces new systematic errors, they are included in the accuracy.

(b) The precision of a celestial frame describes the errors that are surperimposed on the systematic errors and are due to the inaccuracies of the positions and proper motions of stars of the fundamental catalogue (random, regional and, eventually, systematic).

\section{THE PRESENT SITUATION}

Since the appearence of the first modern fundamental catalogue (Auwers Fundamental catalogue), the idea of a purely kinematic definition of the reference frame was never seriously considered. One reason is that stars have sizeable proper motions that have non-random 
components in the velocity space as for instance the solar motion with respect to the nearest stars. But the main reason was that astronomers insisted in having a fundamental system expressed in true equatorial coordinates. This implied that coordinates of stars should contain elements describing the rotation of Earth in Space (precession and nutation) and the motion of the equinox. All the dynamics that are necessary to define a reference plane and an origin using the motion of the Earth are included in this requirement. This is done through the definition of the orbit of the Earth around the Sun and a dynamical representation of the motion of the equator: The formulae giving the position of the true equinox and the true equator as functions of time in a fixed frame give the dynamical definition of the frame as well as the motions of the true equatorial coordinate system.

The methods that are used to refer star positions to the sun or planets have been widely described in the literature (e.g., Woolard and Clemence, 1966). They are essential too for the determination of the equinox in the catalogue. The Sun, various planets and the Moon may be used to determine the equinox correction. The discussion of the results by Fricke (1979) shows a dispersion of $\pm 0^{S} .05$. This leads to an accuracy of about $0^{\prime \prime} .1$ per century for this part of the system. In practice, there are further difficulties in trying to impose a dynamical equinox to the origin of right ascensions of the catalogue and an inconsistency is introduced between the definition and the realization using stars, especially that this offset is correlated with the realization of Ephemeris Time (Duncombe et a1., 1975).

Theoretically, the precession could also be defined dynamically. However, the practical determination of this constant (see Fricke, 1977) is biased by a systematic trend of the stellar proper motions due to the galactic rotation. Kinematic determinations of the constant of precession using a model of galatic rotation are superior to purely dynamical solutions.

The methods used to construct a fundamental star catalogue, namely the FK5, are described elsewhere (see Fricke, 1977 and herein) in detail. Let us simply iterate that in practice, dynamics and kinematics are used jointly or independently to yield the best possible results. The choice between the two approaches is based only on the expected quality of the result and not on conceptual considerations. The hybrid character of the present celestial system of reference is based on a deep comparative discussion of all the existing observations.

This pragmatic approach has permitted the ultimate accuracy possible with the presently available observations: something of the order of $0^{\prime \prime} .15$ per century for the FK5 system.

\section{PROSPECTS FOR IMPROVEMENT IN ACCURACY}

The two dominant limitations in accuracy of the FK5 are the present 
difficulty in having an accurate independent dynamical determination of the constant of precession and the limited precision of the observations of the Sun and planets as referred to stars. So it is difficult to imagine major improvements of the FK5 system without using completely new types of data, some of which actually will become available in the nearest future.

If we consider a system based on a dynamical definition, the most drastic improvement in the knowledge of the motion of a celestial body concerns the Moon, thanks to the ten year series of lunar laser observations. The motion of a fixed point near the center of mass of the Moon is now described to better than $40 \mathrm{~cm}$ in radius vector, corresponding to $0^{\prime \prime} .001$ or $0 " .002$ in longitude in an adhoc dynamical reference frame for more than 10 years. This is already better than the accuracy of the present celestial frames. However, until now, there has been no explicit attempt to use the lunar laser data to improve the system (Mulholland, 1980). But even if we assume that this is done, the main difficulty is not to lose this accuracy when constructing the catalogue. The most critical point lies in the knowledge of the lunar limb (see Froeschlé and Meyer, herein) where the present errors are of the order of 0 ". 3 and also in the $i 11$ determined systematic offset of the center of the apparent figure of the Moon and its center of mass. Another difficulty lies in the fact that only zodiacal stars are occulted and that there are not many such stars in the current fundamental catalogues. So, if the future discussions of lunar laser data may improve significantly parameters used in a classical determination of a celestial reference system, the prospects are much worse if one tries to imagine a completely new type of method to build a celestial reference system based uniquely on the motion of the Moon. A very large number of very precise occultation observations, accurately reduced with improved limb corrections, are necessary before any such attempt is made.

If, now, we turn to a kinematic definition of the system, a drastic improvement comes from the observation of selected extra-galactic radio-sources by VLBI. These sources are used as fiducial points of a fixed celestial reference frame. This has been advocated for many years (Counselman, 1976 or Elsmore, 1979) and several theoretical analyses of the possible improvements were made (Gubanov and Kumkova, 1979 or Wa1ter, 1978). These discussions know that it is necessary to show very precisely the position of the equator in the system, since the observations of declination are referred to the instantaneous axis of rotation of the Earth. Furthermore, only relative right ascensions are obtained and this raises again the problem of an independent determination of the equinox. This can be done using artificial radio-emittors on the Moon or on space-probes. A more appealing method was proposed by Counselman and Shapiro (1968): In addition to the determination of the true instantaneous declination of a pulsar by VLBI, the ecliptic longitude and latitude may be derived from the analysis of the delays of the pulses while the Earth proceeds on its orbit around the Sun. The precision of this method is now better than 0". 1 (Cole, 1976) but does not yet reach the accuracy that is necessary to improve the existing system. 
In an alternate approach, the procedure is reversed and VLBI is used to provide an independent, purely kinematic - actually geometric reference frame without any reference to the Earth motions. Conceptual$1 y$, the only physical assumption is the absence of a transverse component in the cosmological expansion. Krasinsky (1975) has shown that it is possible to construct a fixed coordinate system to an accuracy of the order of the precision of individual VLBI observations. This is done using the mutual angular distances between the sources that are deduced from the observed declinations and relative right ascensions. The frame of reference deduced from the coordinates of fixed objects is also fixed. The axes may have any direction and be totally independent of any direction linked with the Earth or any other body. The accuracy is independent of time: There is no degradation due to the proper motions, and accumulation of observations can only improve it.

The problem with such a system is that the defining objects cannot serve as a materialization of the system for any observational technique except radio. The number of extragalactic sources that are sufficiently point-like and stable to be used for astrometry is rather small: Around 150 (Elsmore, 1979) and their optical counterparts are not a1ways well defined. In any case, they are all very faint and it is necessary to determine their positions with respect to stars that can be candidates for a fundamental catalogue by independent optical measurements. Several such programs have been performed or are in progress (e.g., Walter and West, 1980 or Argue et a1., 1979). But the main part of the task, namely the construction of the system, is not yet undertaken.

Let us assume that such a VLBI reference system exists and let us call $B_{v}\left(\vec{e}_{1}, \vec{e}_{2}, \vec{e}_{3}\right)$ the base of the corresponding coordinate system. The problem is how to extend it to a conventional celestial system containing many stars. Three possibilities may be described.

1. A first procedure consists in determining the positions of the basic sources and their apparent variations with time in a preliminary system (e.g. FK4) defined by a base $B_{p}(t)\left\{e^{\prime}(t), e^{\prime}{ }_{2}(t), e_{3}^{\prime}(t)\right\}$ which may move with time. The programs that are under way will provide such positions. Once these are determined, one imposes to the system $\mathrm{B}_{\mathrm{p}} \mathrm{a}$ fixed rotation $R$ and a rate $R^{\prime}$ such that one has

$$
B_{v}=B_{p}(t) \cdot\left(R+R^{\prime} t\right) .
$$

Applying this transformation to the positions of radio-sources in both systems will permit the determination of $R$ and $R^{\prime}$ and therefore, transform the catalogue in the base $B_{p}$ into a catalogue in the base $B_{v}$.

Of course, the practical application of this procedure will not prove to be so simple because of the errors introduced in the determination of optical sources and errors may appear that would increase the existing ones in the materialization of the preliminary system. It may be necessary to introduce a procedure analogous to that proposed 
by Gubanov and Kumikova (1979), but this will degrade the accuracy of the procedure. As a matter of fact, if a precision of only $0 " .1$ is achieved in connecting stars to extragalactic sources photographically, it is to be expected that the final accuracy will be degraded to the same order of magnitude. This could only be overcome using much more precise determinations of the relative positions of stars and radiosources. Such a possibility will exist with the astrometric use of the space telescope (Van Altena et a1., 1974 and Jefferys, 1979). However, strong difficulties arise from the limited field of view (70 square arc-minutes) and from the fact that the lower limiting magnitude is too high $(m=10$ or 11$)$. As pointed out by C. A. Murray during a discussion, there is at least one AGK3 star within 15' of most sources, so that it is possible to tie a stellar system to VLBI or visual galactic system.

2. Another method that has been proposed (Kovalevsky, 1975, and 1979) uses as an intermediary a terrestrial reference frame. If we assume that the Earth rotation is continuously determined in two different celestial systems, for instance using classical astronomical instruments (base $B_{p}$ ) and VLBI (base $B_{v}$ ), if both systems were perfect, the observed rotation of the Earth in both references should be the same. If this is not the case, the difference represents the offset and the rotation of $B_{p}$ with respect to $B_{v}$.

3. A third method implies the use of radio-stars. There exist a certain number of stars that are known to be radio-emitters and that are brighter than magnitude 10 so that they can easily be incorporated in a fundamental catalogue (Walter, 1977; Débarbat, herein; or Robertson, herein). One hundred such sources well distributed throughout the sky will ideally serve the purpose. Unfortunately, despite the improvement of the radio techniques, we are far from reaching this number (Walter's list has 25 radio-stars with $m<11$ and a few more candidates). So it is necessary to start a specific search for radio-stars. This has not been done because of the mediocre return in astrophysics, but such a programme would be very important for astrometry.

Assuming that these radio-stars are observed by VLBI at two epochs, their positions and proper motions will be obtained in the $\mathrm{B}_{\mathrm{V}}$ system, possibly to better than 0".00l per year. Comparing them with the proper motions of these stars in the $B_{p}$ system, we can express the differences in terms of an offset and a rotation of $B_{p}$ with respect to $B_{v}$.

\section{IMPROVEMENT IN PRECISION}

If we now turn towards precision, two factors play a dominant role in degrading the stellar materialization of celestial reference systems.

1. Random errors in positions and proper motions of stars evident1y reflect in the errors of realization of the system by these stars. Presently, in the FK4, the precision is of the order of 0 ".08 and will probably be reduced to $0 " .04$ in the FK5. 
2. The density of stars belonging to the fundamental catalogue also reflects itself in the practical realization of the system in a given portion of the sky. If, for some uses, the 1515 stars of the FK4 may suffice (polar motion and Earth rotation with astrolabes), in most cases, the fundamental catalogue has to be densified and this usually done at the cost of a severe degradation of the precision. If this may not be the case in some very specific instances (PZT catalogues), usual$1 \mathrm{y}$, one cannot expect a precision better than $0^{\prime \prime} .25$ and sometimes $0 " .4$ (AGK 3 or SAO) probab1y a1so in conjunction with some degradation of the accuracy. In other terms, whenever the direct use of FK4 stars is not possible, an important blur is introduced by the secondary catalogue random and regional errors, preventing to reach also the full accuracy of the system.

The introduction of the FK5 system and catalogue will improve the situation, since the number of stars is tripled. However, this will not solve the difficulty for many purposes, like providing stars to photographic astrometry or a list of candidates for an occultation programme.

A very good remedy is expected to be provided in the future by the astrometric satellite Hipparcos (ESA, 1979). The programme is due to start next year, the satellite being launched in 1986. Three years of observations should provide the positions and proper motions of 100,000 stars with a precision of respectively $0 " .002$ and $0 " .002$ per year. In principle, all bright stars up to photographic magnitude 8.5 will be included together with many fainter stars, especially in the high galactic and ecliptic latitudes. The system of the Hipparcos catalogue will not be inertial. The internal consistency of observations should avoid any detectable regional error, but the origin of the axis will have to be defined a priori and a residual rotation of the system is unavoidable. However, the system may be linked to a VLBI system by one of the procedures described in the last section, preferably by the third one. So we may expect that the Hipparcos catalogue will indeed represent a geometrically fixed celestial sphere with an accuracy of $0 " .002$ and a slowly degrading precision, because of the accumulation with time of random errors in the proper motions.

The problem of the origin and the direction of the axes remains open. For historical reasons, it may be near-equatorial. But by no means, should it be linked to any Earth motion parameter. The fixed Hipparcos system must be used as a reference for all motions and the formulae for the computation of what are called "true positions" should not be part of the system.

\section{FURTHER EXTENSIONS}

The Hipparcos system will be a drastic improvement to the present situation and to the expected situation after the FK5 will become available. Later, it is still possible to imagine further improvement in 
accuracy by repeated observations by VLBI of already known and also new radio-sources that may be discovered among bright stars. Furthermore, a great improvement in accuracy and precision would be provided by an eventual second launch of an astrometric satellite similar to Hipparcos.

The star distribution in the Hipparcos catalogue should be sufficient for many applications, all astronomical techniques for the determination of polar motion and Earth rotation (astrolabes, PZT, zenith telescopes), lunar occultations or reference stars for the observations of major planets. However, the number of reference stars for photographic astrometry must exceed two stars per square degree as provided by Hipparcos. Often also, in order to avoid important magnitude effects, it is necessary to have very faint reference stars with magnitudes comparable to the magnitude of the object to be measured. It will, therefore, be necessary to extend the Hipparcos catalogue to many more stars of higher magnitudes.

This situation will not be new and similar needs already exist now. The extended use of Schmidt telescopes for the measurement of accurate positions of very faint objects (optical counterparts of radio-sources, minor planets, satellites) makes it necessary to use reference stars with magnitudes in the range 10-15. The astrographic catalogue is much too inaccurate to provide good positions and the stars of present reference catalogues (AGK 3, SAO, Yale zones) are too bright. So, their extension to fainter magnitudes is already necessary. The only but very important difference with the post-Hipparcos situation is that the extension will now be based on very imprecise catalogues that are already mediocre extensions of the fundamental catalogue, while later, we shall be able to start directly from the fundamental catalogue to build the extension. There will be one imprecise intermediary less.

Automatic photoelectric meridian circles seem to be now the best instruments for a precise extension and densification of catalogues. These instruments are now entering an operational stage. They may be quite precise, with observational error of the order of 0 ". 10 up to magnitude 13 (Requième, 1979). They also may be very efficient, since it is expected that the Carlsberg automatic meridian circle will observe 100000 stars per year with a precision of $0 " .20$ (Fogh 01 sen and Helmer, 1979). It is possible to expect that future instruments will combine both precision and rapidity. The construction of an extension to the Hipparcos catalogue including half a million stars to a precision of $0^{\prime \prime} .10-0 " .15$ and $0 " .015$ per year is feasable and would suffice for practically all needs of photographic astrometry using classical astrograph or Schmidt telescopes. Because of the need of two epochs separated by at least ten years, it is desirable that observations start soon and that the Carlsberg meridian in the Canary Island not be the only instrument to perform this type of observations. 


\section{CONCLUSION}

While the classical constructions of celestial reference frames are expected to provide sizeable improvement over the presently existing FK4 system, the advent of new observing techniques like VLBI and the astrometric satellite makes us expect, for the early nineties, a drastic improvement of a celestial reference frame in accuracy, precision and density of materialization by stars. In parallel, an effort of further densification to fainter magnitudes is also starting using automatic photoelectric meridians. All these new developments will contribute to make the forthcoming decade very important in the construction of better celestial reference frames.

\section{ACKNOWLEDGMENT}

I thank Drs. Meyer and Mulholland for having very carefully read the manuscript and for their useful remarks.

\section{REFERENCES}

Argue, A. N., Clements, E. D., Harvey, G. M., Murray, C. A.: 1979, in "Modern Astrometry", IAU Col1. 48, F. V. Prochazka and R. H. Tucker ed., p. 155 .

Cole, T. W.: 1976, The Observatory, 96, p. 244.

Counselman, C. C.: 1976, Annual Rev. Astron. and Astroph., 14, p. 197.

Counselman, C. C. and Shapiro, I. I.: 1968, Science, 162, p. 352.

Duncombe, R. L., Seidelman, P. K., Van Flandern, T. C.: 1975, in

"Reference Coordinate Systems for Earth Dynamics", IAU Coll. 26 , Torùn, p. 223.

E1smore, B.: 1979, in "Modern Astrometry", IAU Co11. 48, F. V. Prochazka and R. H. Tucker ed., p. 93.

ESA: 1979, "HIPPARCOS, a Phase A Study", European Space Agency document SCI(79) 10, Paris.

Fogh 01sen, H. J. and Helmer, L.: 1979, in "Modern Astrometry", IAU Coll. 48, F. V. Prochazka and R. H. Tucker ed., p. 219.

Fricke, W.: 1977, Veröff. Astron. Rechen Instituit, Heidelberg, No. 28.

Fricke, W.: 1979, in "Dynamics of the Solar System", IAU Symposium No. 81, R. L. Duncombe ed., p. 133.

Gubanov, V. S. and Kumkova, I. I.: 1979, in "Modern Astrometry", IAU Coll. 48, F. V. Prochazka and R. H. Tucker ed., p. 135.

Jefferys, W. H.: 1979, in "European Satellite Astrometry", C. Barbieri and P. L. Bernacca ed., Padova, p. 111.

Kovalevsky, J.: 1975a, in "Space Astrometry", ESRO Symposium, Frascati, October, 1974, Ref. ESRO SP-108, March, 1975.

Kovalevsky, J.: 1975b, in "Reference Coordinate Systems for Earth Dynamics", IAU Col1. 26, Torùn, p. 123.

Kovalevsky, J.: 1979, in "Time and the Earth's Rotation", IAU Symposium No. 82, D. D. Mc Carthy and J. D. M. Pilkington ed., p. 151. 
Krasinsky, C. A.: 1975, in "Reference Coordinate Systems for Earth Dynamics", IAU Co11. 26, Torùn, p. 381.

Mulholland, J. D.: 1977, in "Scientific Applications of Lunar Laser Ranging", Reide1 Pub1. Co., J. D. Mulholland ed., p. 9.

Mulholland, J. D.: 1980, Reviews of Geophysics and Space Physics, in print.

Requième, Y.: 1979, in "Modern Astrometry", IAU Col1. 48, F. V. Prochazka and R. H. Tucker ed., p. 227.

Van Altena, W. F., Franz, O. G., Fredrick, L. W.: 1974, in "New Problems in Astrometry", IAU Symposium No. 61, W. Gliese, C. A. Murray and R. H. Tucker ed., p. 283.

Walter, H. G.: 1977, Astron. and Astroph., Supp1. 30, p. 381.

Walter, H. G.: 1978, Mitt. Astron. Geselshaft, 43, p. 202.

Walter, H. G. and West, R. M.: 1980, Astron. and Astroph., 86, p. 1 .

Woolard, E. W. and Clemence, G. M.: 1966, "Spherical Astronomy", Academic Press, New York. 